\title{
Research on the Detection and Tracking of Moving Target based on Kernel Method
}

\author{
Huanhai Yang \\ Shandong Institute of Business and Technology, Yantai, Shandong 264005, China
}

\begin{abstract}
The research work in this paper is in the field, the moving target detection spatiotemporal correlation and difference contour tracking algorithm based on a fixed background. The algorithm in the background under the condition of fixed to pay a smaller time complexity, the target detection and tracking has a good effect, so it has higher application value. This paper mainly focuses on the study of motion estimation and compensation algorithm to eliminate the temporal redundancy. Algorithm of detection and tracking of video moving object is a core subject in computer vision field, but also the key technology of intelligent video surveillance system. It combines the research achievements of artificial intelligence and other fields of pattern recognition, image processing, has been widely used in every field of security monitoring, intelligent weapons, video conference, video retrieval. Therefore, detection and tracking algorithm research has the extremely important theory significance and practical value. The starting point of this article is the subjective quality of image reconstruction of how to improve the accuracy of motion estimation and compensation after, to reduce the computation complexity of motion estimation algorithms, to improve the efficiency of motion estimation. This paper makes some studies on the redundant wavelet domain block matching motion estimation and compensation, then the video image for nontranslational motion, the DT triangular mesh motion estimation and compensation in the redundant wavelet domain to do related research.
\end{abstract}

Keywords: Reconstruction, Adaptive, active, pattern recognition, image features

\section{Introduction}

The detection and tracking of video moving object is a core subject in computer vision field, but also the key technology of intelligent video surveillance system [1]. Video moving object detection and tracking is a video image signal by using visible light image sensor or a thermal infrared, low light level imaging sensor uptake of the moving target, after the corresponding digital image processing, detection, extraction of moving targets in video target tracking, and then based on the technology of image feature of the target. The detection and tracking of moving targets two processes are closely related, detection is the basis of tracking, and tracking is to obtain the target motion parameters, such as position, velocity and trajectory, for the subsequent motion analysis, understanding the motion of the target behavior and to provide reliable data source to complete a higher level mission, and provide help for moving target detection [2].

Motion analysis is quite related to pattern recognition, image processing, computer vision, artificial intelligence and other disciplines of knowledge. At the same time, the fast segmentation of moving objects in dynamic scenes, between the non-rigid motion object occlusion or stop treatment for motion analysis has brought some challenges [3]. The fast segmentation of moving objects in dynamic scenes was between the non-rigid motion object occlusion or stop treatment for motion analysis has brought some challenges [4, 5]. In addition, it also widely used in virtual reality environment, simulation training, automatic navigation, robot target acquisition [6]. With the rapid 
development of the technology of image processing, pattern recognition, artificial intelligence and other related fields and a significant improvement of computer performance, in recent years the video moving object detection and tracking technology has been widely used in various fields of social life, economy, military etc.

Behavior understanding can be considered to be time-varying data classification problem, which matching reference sequence to test sequence $[7,8]$. Therefore, the key problem is to obtain the reference behavior understanding behavior from the learning sample sequence, learning behavior sequence matching which can handle the space in the category similar movement patterns and time scale of minor changes. Target classification is the purpose of moving objects from the detected moving regions will be needed to study or our interest region extracted [9]. Therefore, in order to further analyze and tracking behavior for a specific target, the correct classification of moving targets is very necessary [10-12]. This is a real-time system, it requires the system to real-time image acquisition, real-time data processing, real-time data storage, including technical difficulties are mainly two: the first is how to complete the processing of large amounts of data in limited time; the second is how to in a complex background (natural disturbance and artificial interference detection) and tracking of moving objects accurately. The experimental results show that, this method can effectively suppress noise in the target detection, moving target and extract the target point more to detect more complete, so it is practical, in the object tracking, not by matching and search based on the traditional.

Video moving object tracking, according to the Mean Shift moving target tracking algorithm for the lack of traditional update algorithm of target model, and the overall target model update algorithm is proposed in this paper the problem of drift model, a selective model update of the Mean Shift algorithm, the core of the algorithm is the computation of the target model of thought of each component of the whole similarity coefficient matching contribution the various components, to update the target model and to selectively according to the value of the. In the Visual C++6.0 software platform, programming the three tracking algorithm using the OpcnCV library function, and the result of program realization analysis. The experiments prove that Mean SlliR algorithm to update the selective model proposed by this paper is more stable and accurate target tracking.

\section{Related Work and Theory Analysis}

\section{A. Optical flow method image and sequence analysis}

Motion estimation is an important work of multi frame video image super-resolution reconstruction in displacement. Video moving object detection algorithm can be divided into two types according to the relationship between the camera and the target: a static camera relative to the target, namely the video moving target detection under the static background; the other is a camera may according to need to follow a target, or by rotating the acquire a larger regional monitoring, detection of video moving object in dynamic background. The reconstructed image quality depends on the degree of accuracy in the estimation of motion parameters [13].

Motion between images can be divided into two categories: global motion and nonglobal motion. Global motion estimation can be used to solve the general affine transformation model, rather than the global motion estimation problem solving approach are numerous, such as methods based on optical flow equation, based on the block matching method, and block matching and optical flow method combination algorithm.

$$
D_{c}\left(x_{1}, x_{2}, t\right)=D\left(x_{1}+v_{1} \Delta t, x_{2}+v_{2} \Delta t, t+\Delta t\right)
$$

Using the differential chain method, it can be expressed as: 


$$
\frac{\partial D_{c}\left(x_{1}, x_{2}, t\right)}{\partial x_{1}} v_{1}\left(x_{1}, x_{2}, t\right)+\frac{\partial D_{c}\left(x_{1}, x_{2}, t\right)}{\partial x_{2}} v_{2}\left(x_{1}, x_{2}, t\right)+\frac{\partial D_{c}\left(x_{1}, x_{2}, \mathrm{t}\right)}{\partial t}=0
$$

Image sequence is also known as dynamic image [13], image sequence which is composed of a series of relative with the given or assumption of the composition. Frame difference moving target detection algorithm to detect the use of the strong correlation of image based on adjacent frames, and gives the adjacent image acquisition time interval can be expressed as follows:

$$
\begin{gathered}
D_{0}(x, y): N\left(m_{0}, s^{2}\right) \\
\sigma_{0}^{2}(x, y)=\frac{1}{M} \sum_{k=0}^{M-1}\left[g_{k}(x, y)-\mu_{0}(x, y)\right]^{2} \\
\mu_{0}(x, y)=\frac{1}{N} \sum_{k=0}^{N-1} f_{k}(x, y)
\end{gathered}
$$

Statistical average method based on the continuous image sequence pixel by pixel statistical average. Inter frame difference algorithm is simple, programming complexity is low, easy to implement: because the interval between the two frames is short. The average values to approximate the background image, both continuous acquisition of $\mathrm{N}$ image cumulative average:

$$
B_{k}=\frac{1}{N}\left(f_{k}+f_{k-1}+\cdots f_{k-N-1}\right)
$$

Where $\mathrm{N}$ was the image frames, motion velocity value, moving target $\mathrm{N}$ and target size $\mathrm{Bk}$, moving faster, can obtain the background with fewer frames, general $\mathrm{N}$ bigger and more beneficial to get more realistic background estimation.

\section{B. Morphological of digital image analysis}

Currently, mathematical morphology had been widely used in digital image processing and machine vision field, forming a unique digital image analysis method and theory [14]. The mathematical morphology has a complete mathematical basis, which used to analysis and processing for morphological [15-17]. The inter frame difference method using two frames of adjacent image difference operation to get the moving object, the algorithm is simple and easy to realize, the adaptability is strong, but when the moving target due to the excessively high background exposure between adjacent frames is large, easy to expose the background detection for moving object detection, which leads to target the ghosting phenomenon. According to the set of mathematical morphology theory developed, morphological operation basically has four kinds: dilation, erosion, opening and closing operations [18].

$$
\begin{aligned}
& G=\left\{\begin{array}{l}
\theta \ldots \ldots \ldots \ldots \ldots \ldots \ldots \ldots \ldots \ldots \ldots \\
2 \pi-\theta \ldots \ldots \ldots \ldots \ldots i f(G \leq B)
\end{array}\right. \\
& \theta=\sin ^{-1} \frac{\frac{1}{2}[(R-G)+(R-B)]}{\left[(R-G)^{2}+(R-B)(G-B)\right]^{\frac{1}{2}}}
\end{aligned}
$$

Change HIS to RGB transformation formula is somewhat different, it depends on the conversion of the point where the color ring.

When $0^{\circ} \leq \mathrm{H} \leq 120^{\circ}$ : 


$$
B=\frac{I}{\sqrt{3}}\left[1+\frac{S \cos (H)}{\cos \left(60^{\circ}-H\right)}\right], B=\frac{I}{\sqrt{3}}(1-S), G=\sqrt{3} I-R-B
$$

When $120^{\circ} \leq \mathrm{H} \leq 240^{\circ}$ :

$$
R=\frac{I}{\sqrt{3}}\left[1+\frac{S \cos \left(H-120^{\circ}\right)}{\cos \left(80^{\circ}-H\right)}\right], R=\frac{I}{\sqrt{3}}(1-S), B=\sqrt{3} I-R-G
$$

When $240^{\circ} \leq \mathrm{H} \leq 360^{\circ}$ :

$$
\begin{gathered}
G=\frac{I}{\sqrt{3}}\left[1+\frac{S \cos \left(H-240^{\circ}\right)}{\cos \left(300^{\circ}-H\right)}\right], G=\frac{I}{\sqrt{3}}(1-S), R=\sqrt{3} I-G-B \\
M S E(i, j)=\frac{1}{M * N} \sum_{m=1}^{M} \sum_{n=1}^{N}[T(m, n)-F(m+i, n+j)] \\
M P C(i, j)=\sum_{m=1}^{M} \sum_{n=1}^{N} N(i, j)
\end{gathered}
$$

In it:

$$
\mathrm{N}(i, j)=\left\{\begin{array}{l}
1 \ldots \ldots \ldots \ldots \ldots . . . \\
0 \ldots \ldots \ldots \ldots \ldots \ldots \text { others }
\end{array}\right.
$$

\section{Image Features Extraction based on Video Motion Nuclear Method}

\section{A. Block Matching Method}

Block matching aimed to find the best motion vector estimation through the pixel domain search procedures. The basic idea is shown in Figure 1, by considered a central location in $U$ frame (the frame) pixel $(\mathrm{m} 1, \mathrm{~m} 2) \mathrm{m} 1 * \mathrm{~m} 2$ block, at the same time, search the $\mathrm{K}+1$ frame (search frame) to obtain the best matching block position the same size, in order to determine the $\mathrm{K}$ frame $(\mathrm{m} 1, \mathrm{~m} 2)$. Considering the displacement from a computational perspective, search is usually limited within the scope of the size of a $\mathrm{M} 1 * \mathrm{M} 2$, the range is called the search window.

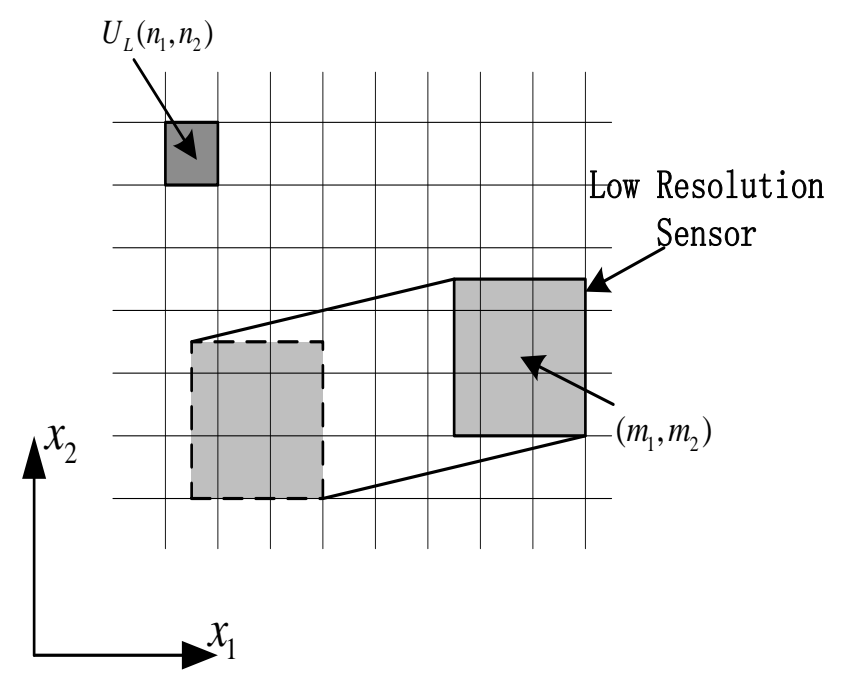

Figure 1. Block Matching Diagram 


\section{B. The correlation of the different block detection}

In a real application scenario, the background is completely still is not possible, especially in outdoor scenes, the background is changing and shows multi peak distribution. For example, when the fan in the rotation, the foliage in the tree swing occurred in such situation, it will repeatedly covering a pixel point and leave, resulting in the pixel values change dramatically, in order to better extract the moving target of interest, should put the swaying branches and leaves, the rotation of the fan is also seen as background. This is not enough to describe the pixel background with a unimodal distribution, therefore uses the multi peak distribution model (such as mixed Gauss background model) to describe the background.

RGB transfer to HIS around is a simple and fast nonlinear transform, the normalized RGB, its mathematical conversion relations are as follows:

$$
\begin{aligned}
& \text { Avgarea }_{m}=\frac{\sum_{k=1}^{N} S_{k}}{N} \\
& Q_{k}(x, y)= \begin{cases}0 & S_{k}>\text { Avgarea }_{m} \\
1 & S_{k} \leq \text { Avgarea }_{m}\end{cases}
\end{aligned}
$$

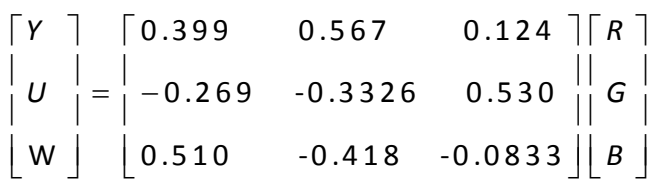

For no matching pixel values of all the Gauss distribution device, a new Gauss distribution corresponding to the minimum weight replacing one Gauss distribution is introduced, the new Gauss distribution to the current pixel is mean, re initialize a larger variance and less weight, a histogram is a variety of spatial processing technology foundation. Histogram operation can be can be through the development of a transform function, this function only depend on the input image histogram information can be automatically image gray level distribution is very narrow into a high contrast and changeable image gray tone, so as to achieve the effect of image enhancement.

\section{Fast adaptive motion estimation and compensation algorithm}

This paper presents a fast adaptive block matching motion estimation and compensation algorithm based on redundant wavelet transform. The algorithm steps are as follows:

(1) The input video image of redundant discrete wavelet transforms wavelet coefficients and the sub-band block.

(2) If the image as the reference frame (I frame), then the decomposed sub-band images are stored and sampling, discrete wavelet transform DWT corresponding to the image, and go to step (5); otherwise.

(3) If the image is predicted frame ( $\mathrm{P}$ frame), using adaptive prediction of initial search point method and fast adaptive block matching motion estimation search algorithm for motion estimation, the best motion vector of image blocks in the redundant wavelet domain to get. According to the error of each candidate vector and the final block motion vector prediction coefficients, each candidate vector method in the corresponding adaptive adjustment.

(4) The motion vectors obtained by the RDWT coefficients and motion estimation of I frames, each frame of $\mathrm{P}$ with image motion compensation prediction image respectively, $\mathrm{P}$ frame in the redundant wavelet domain, and calculate the residual image and the corresponding. Residual image of redundancy in wavelet domain and then down sampled, obtain the residual image based on DWT coefficients. 
Figure 2 was the redundant wavelet transform fast adaptive block matching motion estimation and compensation algorithm based on the flow chart.

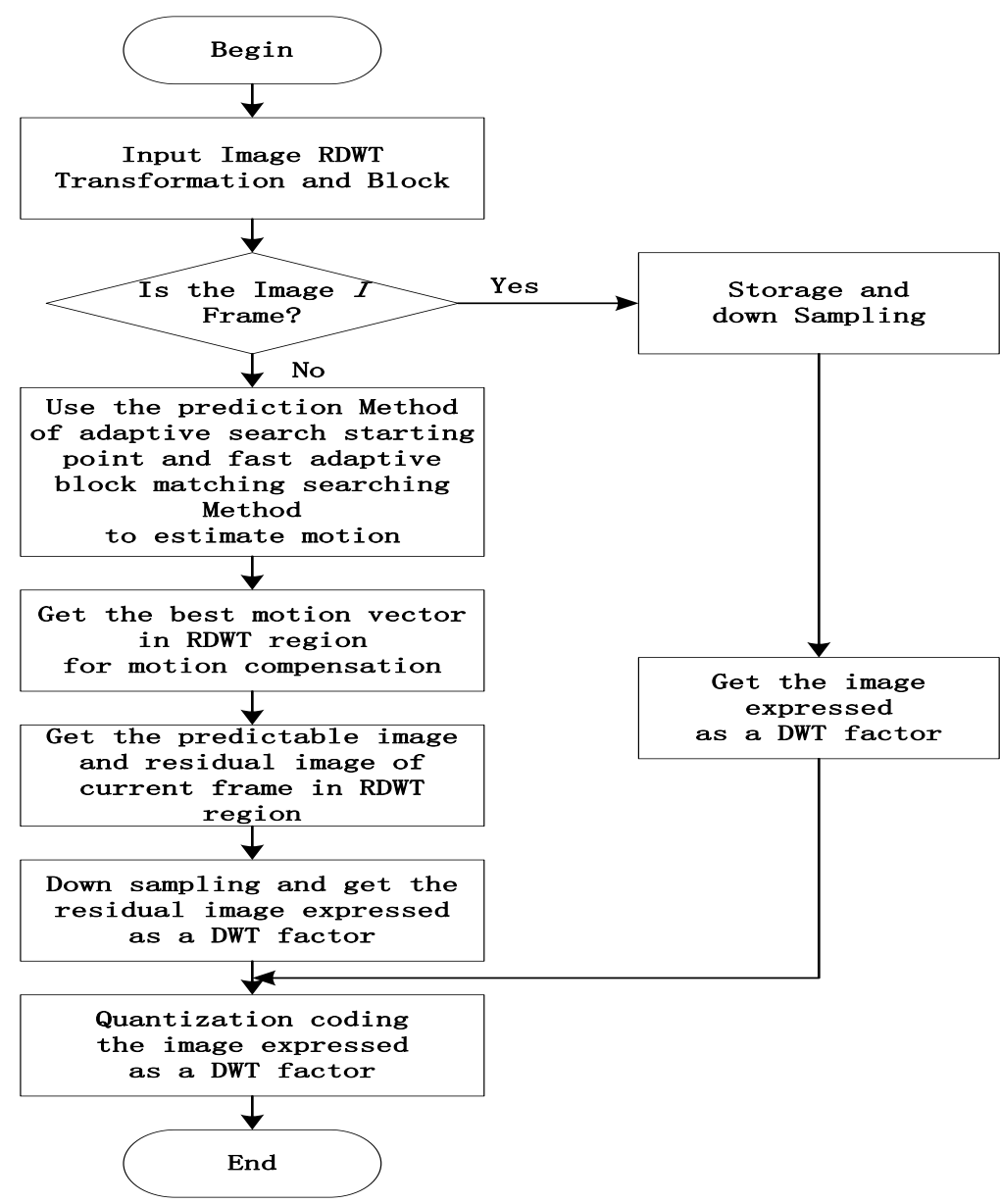
Figure 2. The Redundant Wavelet Transform Fast Adaptive Block Matching
Motion Estimation and Compensation Algorithm

\section{Experimental and Results}

\section{A. Describe the main algorithm experimental model}

The motion detection process method of mixed Gauss background model in background modeling allows moving targets exist, especially suitable for the detection of outdoor light and changes in the weather not too strong cases of small size and fast moving targets, but also can be a good judge of conversion between the background properties of object movement, has a strong adaptive ability. But the mixed Gauss background model of computing complexity and computational complexity with the number of Gauss model increases, must improve the hardware equipment and optimization method to solve the problems in this regard, in addition to the large. The effect of target motion detection of slow speed is not ideal, and the overall brightness of sudden change is very sensitive, sometimes the entire video frame that is prospect.

The double helix classification was usually used to perform the test of pattern recognition algorithms result, the plane coordinate form of double spiral can be used to represent the parameter equation as follows: 


$$
\begin{aligned}
& :\left\{\begin{array}{l}
x_{1}=\left(a_{1} \theta+e_{1}\right) \cos (\theta) \\
y_{1}=\left(a_{1} \theta+e_{1}\right) \sin (\theta)
\end{array}\right. \\
& :\left\{\begin{array}{l}
x_{2}=\left(a_{2} \theta+e_{2}\right) \cos (\theta) \\
y_{2}=\left(a_{2} \theta+e_{2}\right) \sin (\theta)
\end{array}\right.
\end{aligned}
$$

Among them, $\mathrm{a}_{1}, \mathrm{a}_{2}, \mathrm{e}_{1}$ and $\mathrm{e}_{2}$ are parameters to be set. In this experiment, $\theta$ is the random number and interval.

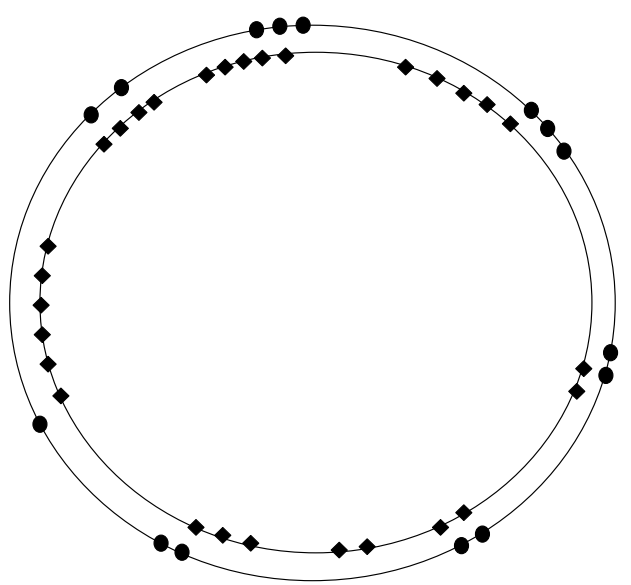
Figure 3. The Unrestricted Conditions of GF Space Model Polynomial
Kernel Classification

\section{B. SIFT algorithm for feature extraction}

Tracking algorithm based on area matching, according to the image regions of the moving objects in the common characteristics of information to track. The main idea of the method search related areas of maximum target area in one frame in the current video frame, also called on relevant type, the specific approach is to target image in the current frame image with different offset displacement, then according to the similarity metric criterion of two images each offset value some overlap, by correlation processing of the current frame image and target image and the target image of the same size, the use of criteria and related processing results, determine the target position in current frame, the greatest similarity of the corresponding position is the movement target position. Each frame of a peak signal to noise ratio (SNR) and each frame for each macro-block average search points (ANSP) respectively is shown in Figure 4. 


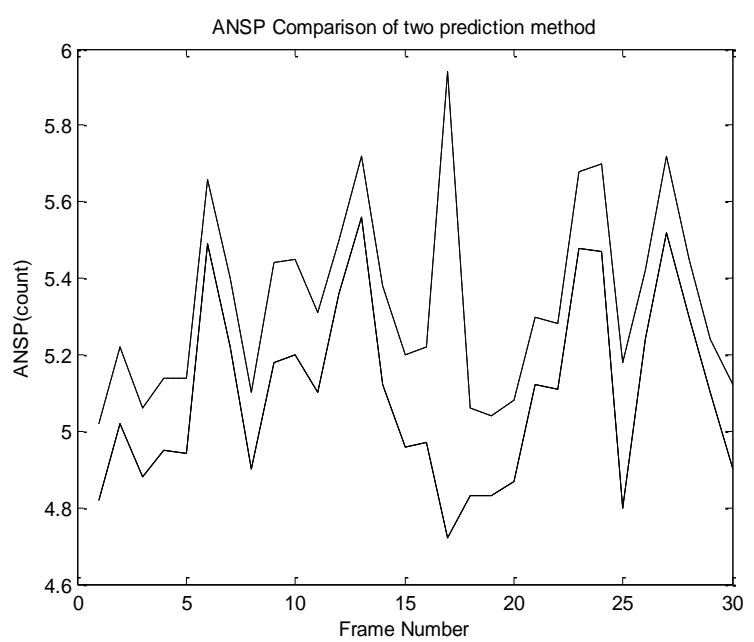

Figure 4. Each Frame of the Peak Signal to Noise Ratio (SNR)

\section{Simulation results and analysis}

Through programming and compare the difference between frame, three frame difference and mixed Gauss background difference method, we can draw the following conclusions: three frame difference method has good adaptability to dynamic environment, but cannot extract the complete moving target; background mixture models of Gauss difference method can extract the background model to exist in the moving object in the scene, and real-time updating background model, changes of the scene has certain adaptability, but when the scene of global lighting change, will the entire video frame for moving target detection, false positive phenomenon. The moving target detection in outdoor scenes was shown in Figure 5.

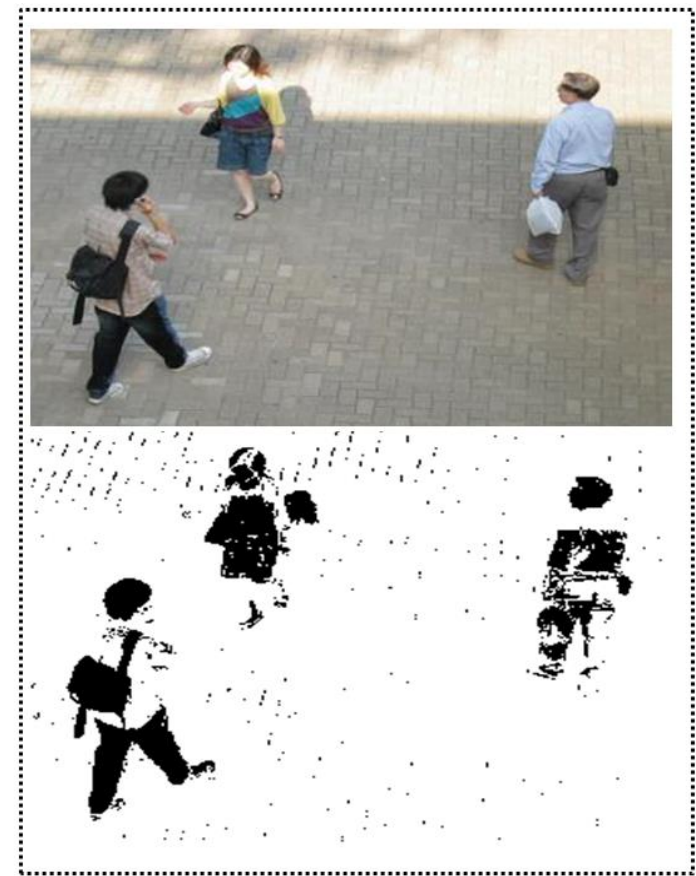

Figure 5. Moving Object Detection in Outdoor Scenes

In the aspect of video moving object detection, introduces three kinds of algorithms at present mainly: frame difference method, background difference method and optical flow 
method; focus on -- three frame differencing method improved inter frame difference method, analysis the advantages and disadvantages of this method; according to the key points of the background difference method is establishing and updating background model, this paper focuses on the modeling method of mixed Gauss model, and the model is based on the background of the advantages and defects in the application of differential method.

Each groups of experimental data is acquired by digital imaging equipment, the image motion parameters according to the actual movement situation to select the appropriate method to calculate the. According to the character of Butterworth filter, in order to better maintain the edge details, select the parameter $\mathrm{D}=0.9, \mathrm{n}=3$, its frequency response as shown in Figure 6:

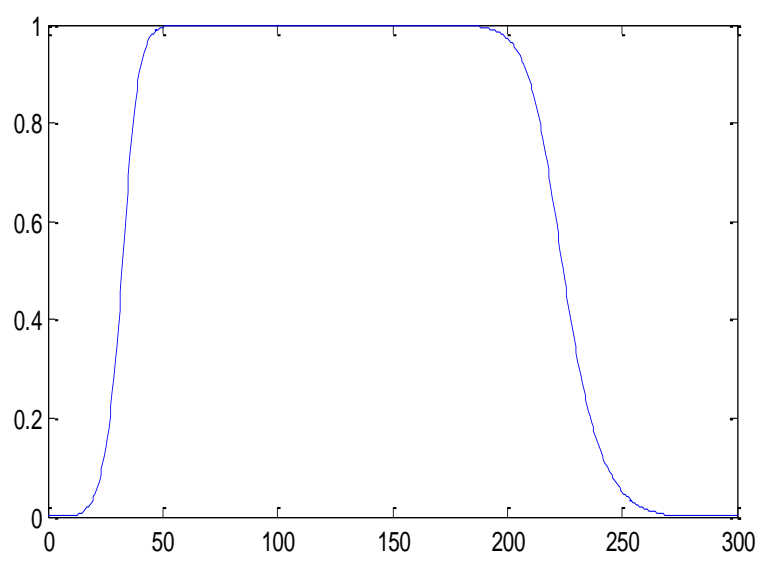

\section{Figure 6. The Six Ordered Butterworth Low-pass Filter Frequency Responses}

\section{Conclusions}

In the tracking of video moving object, summarizes four kinds of commonly used algorithms: Based on tracking algorithm, tracking algorithm based on 3D model, region matching tracking algorithm based on feature matching and tracking algorithm based on active contour; focus on the feature matching tracking algorithm in Mean Shift moving target tracking algorithm, for its lack of target model update algorithm and the overall target model update algorithm model drift problem, tracking algorithm is presented a selective model update Mean Shift moving target; the algorithm is based on matching the size of the contribution of each component object model, to select the target model components that need to be updated, thus realizing the selective target model update.

\section{References}

[1] T. Wang, J. G. Proakis, E. Masry and J. R. Zeidler, "Performance degradation of OFDM systems due to Doppler spreading”, IEEE Transactions on Wireless Communications, vol. 5, (2006), pp. 1422 - 1432.

[2] T. Fusco and M. Tanda, "ML frequency offset and carrier phase estimation in OFDM systems with noncircular transmissions", in Proc. EUSIPCO 2004, (2004), pp. 897-900.

[3] C. Shin, W. Robert Jr. and E. J. Powers, "Blind Channel Estimation for MIMO-OFDM Systems", IEEE Transactions on Vehicular Technology, vol. 56, (2007), pp. 670-685.

[4] C. Dubuc, D. Starks, T. Creasy and Y. Hou, "A MIMO-OFDM prototype for next-generation wireless WANs", IEEE Commun. Mag., vol. 42, no. 12, (2004), pp. 82-87.

[5] A. van Zelst and T. C. W. Schenk, "Implementation of a MIMO OFDM-based wireless LAN system", IEEE Trans. Signal Process., vol. 52, (2004), pp. 483-494.

[6] G. L. Stüber, J. R. Barry, S. W. Mclaughlin, Y. Li, M. A. Ingram and T. G. Pratt, "Broadband MIMOOFDM wireless communications", Proc. IEEE, vol. 92, no. 2, (2004), pp. 271-294. 
[7] W. Xin, Z. -H. Tan and C. Xia, "Doppler diversity receiver for broadband wireless OFDM system under high-speed mobile environments", IEEE International Symposium on Microwave, Antenna, Propagation and EMC Technologies for Wireless Communications, vol. 2, (2005), pp.1444 - 1447.

[8] C. Donghua and Q. Hongbing, "Doubly selective channel estimation for subband and OFDMA using basis expansion models".

[9] Q. H. Spencer, A. L. Swindlehurst and M. Haardt, "Zero-Forcing Methods for Downlink Spatial Multiplexing in Multiuser MIMO Channel”, IEEE Transactions on Signal Processing, vol. 52, no. 2, (2004) May, pp. 461-471.

[10] M. Sadek, A. Tarighat and A. H. Sayed, "A Leakage-based Precoding Scheme for Downlink multi-user MIMO Channels", IEEE Transactions on Wireless Communications, vol. 26, no. 8, (2008), pp. 15051515.

[11] A. Tarighat, M. Sadek and A. H. Sayed, "A multi User Beamforming Scheme for Downlink MIMO Channels based on Maximizing Signal-to-Leakage Ratios", IEEE International Conference on Acoustics, Speech, and Signal Processing, (2005), pp. 1129-1132.

[12] J. Z. Li, J. B. Li and S. F. Shi, "Concepts, Issues and Advance of Sensor Networks and Data Management of Sensor Networks", Journal of Software, vol. 14, no. 10, (2003), pp. 1717-1727.

[13] S. Tilak, N. B. Abu-Ghazaleh and W. Heinzelman, "A Taxonomy of Wireless Micro-sensor Network models", Mobile Computing and Commuications Review, vo. 1, no. 2, (2002), pp. 1-8.

[14] D. Culler, D. Estrin and M. Srivastava, "Overview of Sensor Networks", IEEE Computer Society Officers, vol. 11, no. 10, (2005), pp. 15-25.

[15] T. Matsuo, Y. Kaneko and M. Matano, "Introduction of Intelligent Vehicle Detection Sensors", IEEE/IEEJ/JSAI International Conference on Intelligent Transportation Systems, vol. 5, no. 12, (1999), pp. 709-713,.

[16] J. Deng, R. Han and S. Mishra, "Inrusion tolerance and Anti-traffic Analysis Strategies in Wireless Sensor Networks", IEEE 2004 International Conference on Dependable Systems and Networks, vol. 10, no. 11, (2004), pp. 477-490,

[17] Q. R. Li, L. Y. Wei and S. F. Ma, "The Model Analysis of Vehicles Situation and Distribution in Intersections Abased on Markov Process", IEEE International Conference on Intelligent Transportation Systems, vol. 2, no. 10, (2003), pp.1076-1080,

[18] A. Wadaa, S. Olariu, L. Wilson, M. Eltoweissy and K. Jones, "On Providing Anonymity in Wireless Sensor Networks", 10th International Conference on Parallet and Distributed Systems, vol. 5, no 10, (2004), pp. 125-136.

\section{Author}

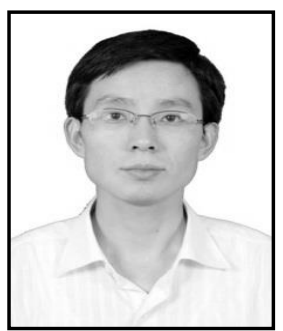

Huanhai Yang, works currently as a computer teacher in Shandong Institute of Business and Technology. He has achieved a master's degree of Computer Application Technology from Naval Aeronautical Engineering Institute. His major interest work area is Machine Learning and Data mining, Information Fusion. 